\title{
TLR4/NF-KB SIGNALING PATHWAY IS A KEY PATHOGENIC EVENT LEADING TO KIDNEY DAMAGE IN UUO INDUCED RENAL FIBROSIS
}

\author{
Amr M. T. Allam ${ }^{1 *}$, Mohamed A. Abdel Aziz ${ }^{1}$, Thorya S. El- Deeb ${ }^{2}$, Abdel-Razik H. \\ Abdel-Razik $^{3}$ and Bakheet E. M. Elsadek ${ }^{1}$ \\ ${ }^{1}$ Department of Biochemistry, Faculty of Pharmacy, Al-Azhar University, Assiut, \\ Egypt \\ ${ }^{2}$ Department of Biochemistry, Faculty of Medicine, Assiut University, Assiut, Egypt \\ ${ }^{3}$ Department of Histology, Faculty of Veterinary Medicine, Beni-Suef University, Beni- \\ Suef, Egypt
}

*Corresponding author: amrallam@azhar.edu.eg

\begin{abstract}
Chronic Kidney Disease (CKD) is a public health problem worldwide. Renal fibrosis is regarded as the final common pathologic manifestation of a wide variety of CKDs. There are many molecules and cells that are associated with progression of renal fibrosis. The toll like receptor (TLR) family serves an important regulatory role in the innate immune system, and recent evidence has implicated TLR signaling in the pro inflammatory response of a variety of endogenous and exogenous stimuli within the kidney. Innate immune activation via TLR4 contributes to acute kidney injuries but its role in tissue remodeling during CKD is unclear. The current study aimed to determine the expression pattern of renal TLR4, nuclear factor kappa B (NF-kB) and alpha-smooth muscle actin ( $\alpha$-SMA) after 3,7 and 14 days of unilateral ureter obstruction (UUO) model in mice, and explore the impact of TLR4 expression on obstruction-induced renal fibrosis and myofibroblast proliferation. We reported an elevated expression of TLR4 and its downstream modulator $\mathrm{NF}-\kappa \mathrm{B}$ in all grades of renal fibrosis of UUO mice. Significant increase in interstitial collagen deposition and myofibroblast activation $(\alpha-$ SMA) were also evident in UUO mice. In conclusion, our findings confirmed previous reports which identified TLR4/NF-kB signaling pathway as a significant mediator of both inflammatory and fibrotic renal injury.
\end{abstract}

Keywords: Toll like receptor; Nuclear factor kappa B; Inflammation; kidney; Unilateral ureteral obstruction; Alpha-smooth muscle actin; Fibrosis. 


\section{Introduction}

Chronic kidney disease (CKD) is a worldwide health problem defined as the presence of kidney damage or decreased estimated glomerular filtration rate. Interstitial inflammation, tubular atrophy, and fibrosis are the common pathological mechanisms of CKD (Yamaguchi et al., 2015). Among these, fibrosis is regarded as the final common pathologic manifestation of a wide variety of CKDs (Webster et al., 2017). The implicated mechanisms of renal fibrosis include infiltration of inflammatory mononuclear cells, interstitial myofibroblast activation with a large proportion of alphasmooth muscle actin ( $\alpha$-SMA) synthesis and an imbalance of components of the extracellular matrix (ECM) (including collagen I, III, and IV and fibronectin) due to their excessive synthesis and reduced breakdown. This process yields irreversible organ scarring and diminished renal function (Zeisberg and Neilson, 2010; Nogueira et al., 2017).

Regardless of the initial insult leading to renal fibrosis, chronic inflammation appears to be a critical process heralding fibrogenesis (Shankar et al., 2011). Induction of various pro-inflammatory cytokines, chemokine and adhesion molecules attracts the transmigration of macrophages and $\mathrm{T}$ cells from the circulation to the interstitium, thereby further enhancing the inflammatory state (Silverstein, 2009). However, the factors that regulate onset, resolution or progression of renal inflammation and fibrosis remain unclear (Liu et al., 2019).

Toll-like receptors (TLRs) are innate immune receptors expressed by immune cells, including macrophages, dendritic cells, lymphocytes, and nonimmune cells including kidney tubular epithelial cells (TECs), endothelial cells and podocytes (Anders, 2010). TLRs recognize pathogen associated molecular patterns present on microorganisms but also recognize endogenous ligands released by damaged or stressed tissues including heat-shock proteins, high-mobility group box 1, fibronectin and biglycan (Vabulas et al., 2002; Schaefer et al., 2005; Yu et al., 2006). Upon activation, TLRs signal via an adaptor molecule MyD88, leading to translocation of a fundamental cellular mediator nuclear factor kappa B (NF-kB) into the nucleus with consequent upregulation of pro-inflammatory cytokines and chemokines, in turn initiating local inflammation and leukocyte accumulation (Mora and Navarro, 2006). Among all TLRs, TLR4 has been reported to be implicated in the pathogenesis of various acute kidney injuries and the occurrence of diabetic nephropathy (Leemans $\boldsymbol{e t}$ al., 2005; Wu et al., 2007; Kaur et al., 2012).

Only few studies have yet addressed the implement of TLR4/NF-kB pathway in the process of tissue remodeling in chronic kidney diseases. Therefore, using an in vivo model of unilateral ureteral obstruction (UUO), we sought to determine the expression of both renal TLR4 and NF-kB after 3, 7 and 14 days of UUO, and explore the impact of TLR4 expression on obstruction-induced renal fibrosis and myofibroblast proliferation. 


\section{Materials and Methods}

\subsection{Animals and experimental design}

Twenty four male balb/c mice (age, 6-8 weeks; weight, 18-22 g) were purchased from the laboratory animal colony, Assiut University, Assiut, Egypt. Mice (6 per cage $)$ were acclimated for 1 week at a regulated environment $\left(25^{\circ} \mathrm{C}, 12 \mathrm{~h}\right.$ light/dark cycle) with free access to standard pellet diet and tap water add libitum. Animal experiments were performed after approval by the Institutional Animal Care and Use Committee of Faculty of Medicine, Assiut University, Assiut, Egypt.

\subsection{UUO Model}

Subsequent to acclimatization, mice were randomly assigned into 4 groups as follow: 3 days ligated group $(n=6), 7$ days ligated group $(n=6), 14$ days ligated group $(n=6)$ and Sham-operated group $(n=6)$. The UUO model was performed under intraperitoneally anesthesia with an anesthetic solution that contained $100 \mathrm{mg} / \mathrm{kg}$ ketamine and $10 \mathrm{mg} / \mathrm{kg}$ xylazine. The anesthetized animal was positioned supine on a dissection plate, heated by artificial lighting, and the abdominal skin was cleaned, shaved and disinfected with iodine alcohol. Following flank incision, left ureter was exposed and UUO was performed aseptically by complete ligation of the left ureter using a 5-0 silk tie suture at two points and cut between the ligatures to prevent retrograde urinary tract infection (Chevalier et al., 2009). The sham-operated group was received the same surgical procedures but without ureter ligation.

After 3, 7 or 14 days of UUO or sham operation (Xu et al., 2013), each animal was housed in an individual metabolic cage for urine collection. The obtained urine samples were collected, centrifuged, and then stored at $-20^{\circ} \mathrm{C}$ for subsequent evaluation of urinary biochemical indices. Blood samples were collected from retro-orbital plexus for serum preparation and the mice were sacrificed by cervical decapitation under ketamine/xylazine anesthesia, autopsy was performed and renal tissues were harvested and immediately washed with physiological saline. The excised kidney was divided into 3 parts; one part was stored in 10\% neutral buffered formalin solution and subjected for histopathological examination. The other two parts were kept in RNA later solution and stored separately at $-80{ }^{\circ} \mathrm{C}$ for subsequent biochemical and molecular assays.

\subsection{Histopathological examinations of renal tissue}

For histopathological examination, kidney tissues were fixed in $10 \%$ neutral buffered formalin solution. Washing was carried out in sterile tap water, and then in serial dilutions of alcohols (methyl, ethyl and absolute ethyl) which were used for dehydration. Specimens were cleared in xylene and embedded in paraffin at $56{ }^{\circ} \mathrm{C}$ in a hot air oven for 24 hours. Paraffin bees wax tissue blocks were prepared for sectioning at 4 microns thickness by a sledge microtome and the obtained tissue sections were placed on glass slides, deparaffinized, stained by hematoxyline \& eosin (H\&E) stain and Masson's trichrome (MTC) and then examined under light microscope (Olympus, USA) by a histopathologist who was blinded to the groups arrangement for assessment of histopathological changes (Page, 1983). The percentage of area occupied by Masson's staining was measured in six separate microscopic fields in each slide using a digital 
imaging software program (Image $\mathbf{J}^{\circledR}$ software, National Institutes of Health, Bethesda, USA), and the mean area for each slide was obtained, then the means \pm SEM was calculated for each group.

\subsection{Assessment of renal performance indices}

Serum creatinine was assayed by kinetic procedure (Peake and Whiting, 2006) using a kit provided from Human Diagnostic (Wiesbaden, Germany). Blood urea nitrogen (BUN) was assayed by colorimetric procedure (Tabacco et al., 1979) using a kit provided from Biomerieux Sa (Lyon, France). Microalbuminuria was estimated by turbidimetrical procedure (Harmoinen et al., 1987) using a kit provided from BioSystems (Barcelona, Spain).

\subsection{Western blot}

Kidney tissue homogenates were prepared in ice-cold Tris-HCl lysis buffer, $\mathrm{pH}$ 7.4 containing $1 \%$ protease inhibitor cocktail (Cell Signaling Technology, Inc., MA, USA) using Potter-Elvehjem rotor-stator homogenizer, fitted with a Teflon pestle (Omni International, Kennesaw, GA, U.S.A). Proteins in each corresponding homogenate were denatured at $95{ }^{\circ} \mathrm{C}$ for 5 minutes in $2 x$ Laemmli buffer followed by addition of 5\% 2-mercaptoethanol. SDS-PAGE electrophoresis was achieved by loading $50 \mu \mathrm{g}$ protein per lane at 75 volts through $12 \%$ resolving gel followed by 125 volts during approximately 2 hours and transferred to a PVDF membrane using T-77 ECL semidry transfer unit (Amersham BioSciences UK Ltd) for 2 hours. Immunoblotting was carried out by incubating the PVDF membrane in TBS buffer containing $0.1 \%$ Tween and $5 \%$ defatted milk for one hour at $4{ }^{\circ} \mathrm{C}$, followed by overnight incubation at $4^{\circ} \mathrm{C}$ with mouse monoclonal primary antibodies TLR4, NF-kB and $\alpha$-SMA (Santa Cruz Biotechnology, Inc., CA, USA) at 1:1000 dilution. After being washed three times with TBST buffer, each membrane was incubated for 1 hour at room temperature with an alkaline phosphatase-conjugated goat anti-mouse secondary antibody (Novus Biologicals, LLC, Littleton, CO, USA) at a dilution of 1:5000. After being washed four times with TBST, the membrane bound antibody was detected with a commercially available BCIP/NBT substrate detection Kit (Genemed Biotechnologies, Inc., CA, USA). Equivalent protein loading for each lane was confirmed by stripping and re-blotting each membrane at $4{ }^{\circ} \mathrm{C}$ against mouse monoclonal anti GAPDH antibody (Santa Cruz Biotechnology, Inc., CA, USA) at a dilution of 1:5000. The analysis was repeated to assure reproducibility of results. The density of each band is determined using a digital imaging software program (Image $\mathbf{J}^{\circledR}$ software, National Institutes of Health, Bethesda, USA) and expressed as a percentage of GAPDH density.

\subsection{Statistical analysis}

Statistical analyses of the data were carried out using GraphPad prism version 5.0 (Graph pad software San Diego, USA). Data comparisons were performed using analysis of variance (ANOVA) followed by Tukey's t-test. The levels of significance were accepted with $\mathrm{p}<0.05$ and all relevant results were graphically displayed as mean \pm SEM. 


\section{Results}

\subsection{Mortality rate, morphological changes and body weight of UUO mice}

In the current study, all animals in the four groups remained alive throughout the experimental period except for a single mortality which was recorded in the UUO 14d group on day 8 . We have also observed lowered daily food intake and a decreased in physical activity indicated by decreased responses to external stimuli in UUO mice after the operation. These symptoms began to disappear gradually after the mice recovered from the surgery. UUO mice showed a temporary decrease in body weight. Shortly after that, they started to regain weight again with smaller rate compared to sham group (Figure 1).

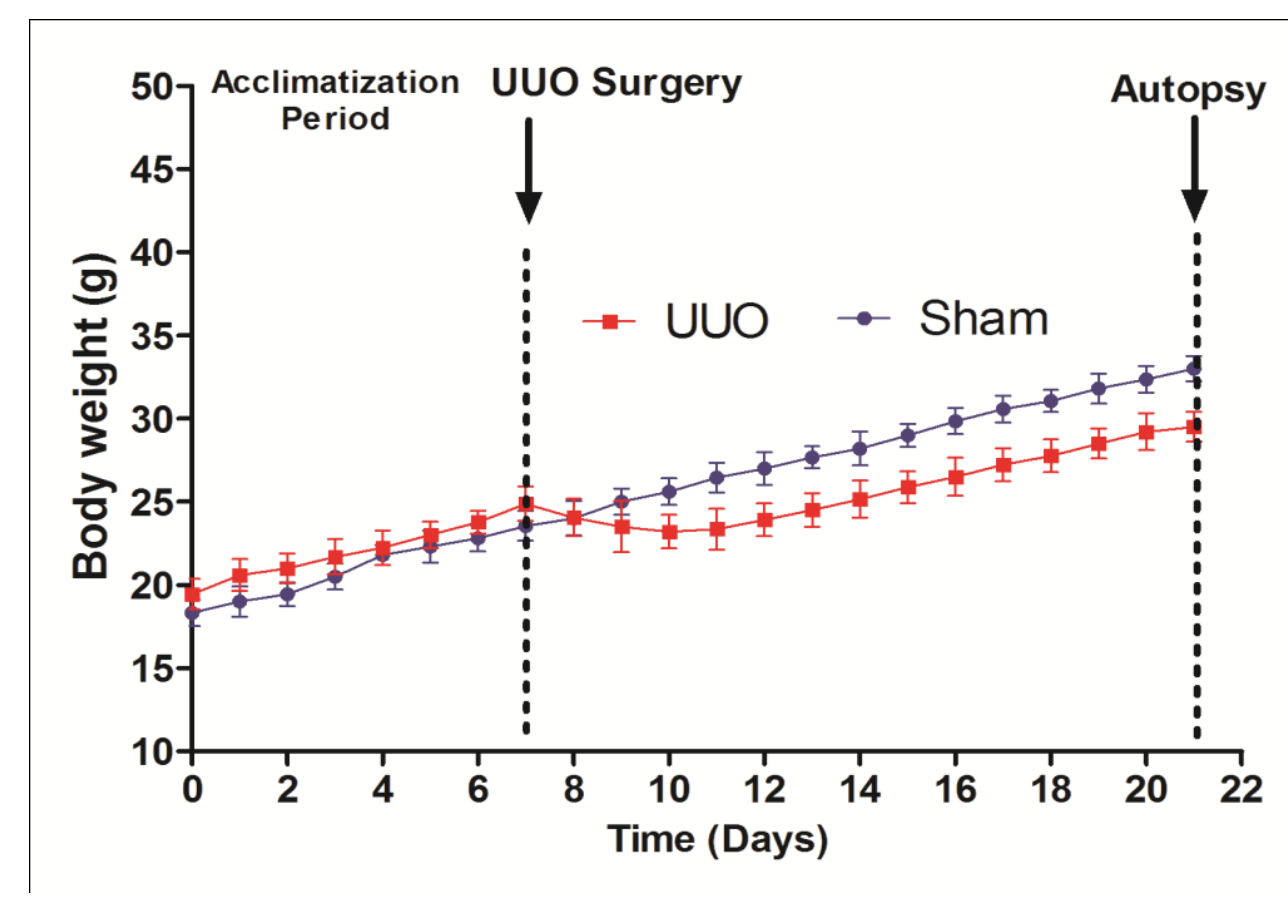

Figure 1: The UUO effect on change in the animal body weight during the experimental period.

\subsection{Histopathological assessment.}

Compared to the sham operated group, it was found that the kidneys from the UUO ligated side were swollen, turbid fluid, and considerably renal parenchyma thinning (Figure 2). Microscopic examination showed the representative H\&E and MTC stained sections of kidney from UUO and control mice. No significant changes were observed in the tubulointerstitium of sham treated mice (Figure 3A), while UUO mice showed clear damages in a time-dependent manner. By day 3, significant tubulointerstitial injuries occurred including tubular dilation, tubular atrophy, few degenerated renal corpuscles and dilation of capillaries in the medulla. On postoperative days 7 and 14, more severe interstitial changes occurred including cortical thinning, further tubular dilation, renal papillary capillary expansion, inflammatory cell infiltration, increased number of fibroblasts, and interstitial expansion. The histopathological changes were scored as follows: normal appearance (0), $25 \%$ change 
(1), 26-50\% change (2), 51-75\% change (3), and 76-100\% change (4) as illustrated in figure $4 \mathrm{~A}$.

Additionally, MTC staining showed fine collagenic fibers between the renal tubules and around the blood vessels of sham mice (Figure 3B). On the other hand, Masson's staining showed increased collagen fiber condensation between renal tubules in a time-dependent manner of UUO mice. Massive condensation of collagen fiber was observed in UUO 14d group indicating an excessive fibrotic renal damage (Figure 3B).

Sham

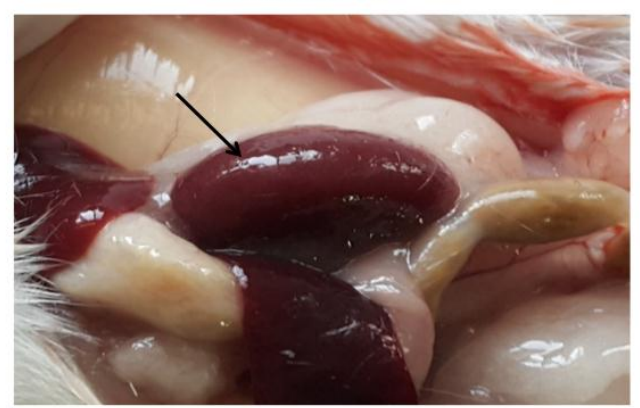

UUO 7d

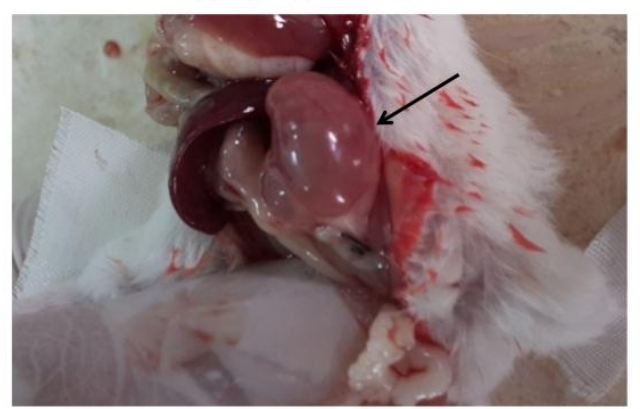

UUO 3d

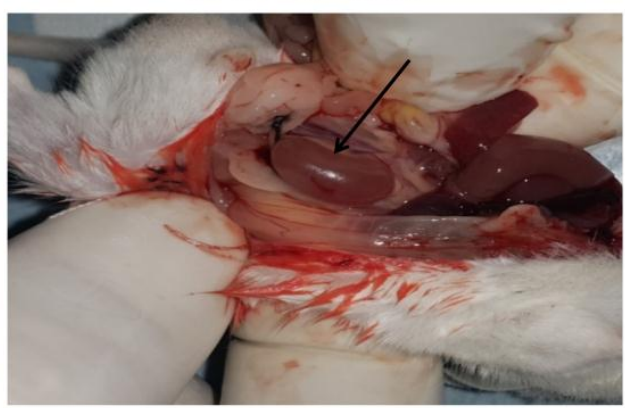

UUO 14d

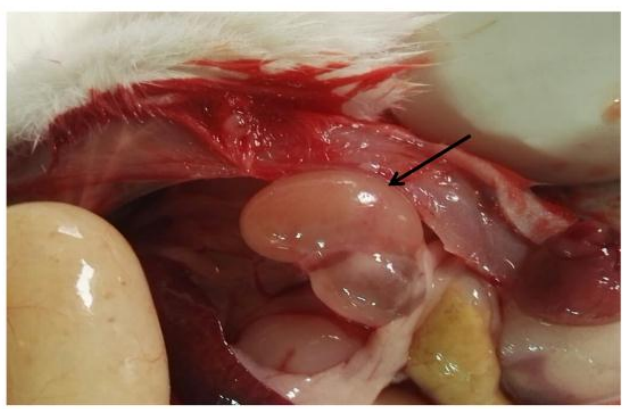

Figure 2: Gross appearance of the mice kidneys in sham operated group and after unilateral ureteral obstruction (UUO). Representative appearance of the left obstructed kidneys shows a swollen enlarged kidney which filled with turbid brown urine. 


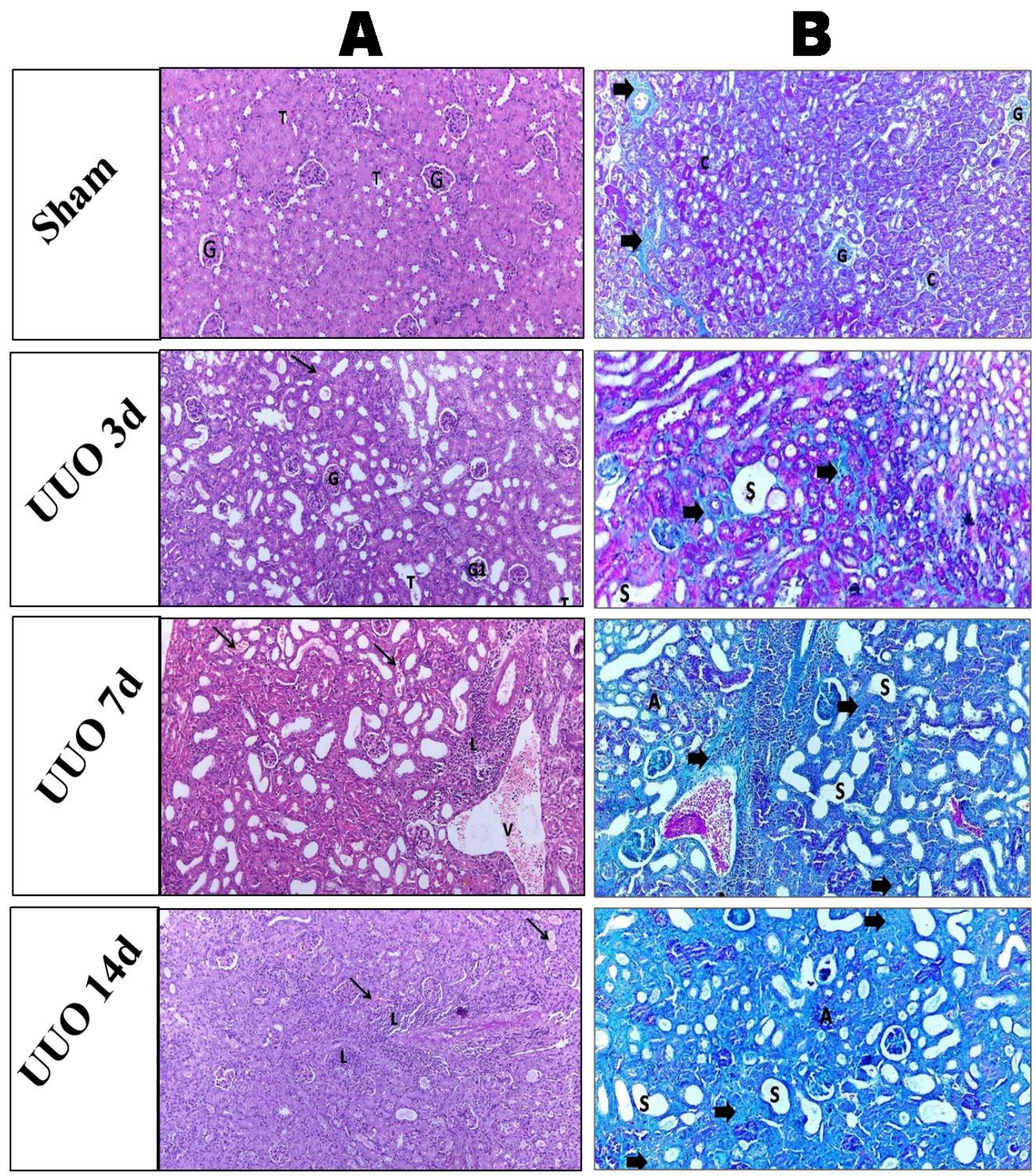

Figure 3: Representative Photomicrographs of renal sections stained by H\&E (Fig. 3A) and Masson's trichrome staining (Fig. 3B) (X10). Kidney tissues in the sham group (Fig. 3A) showed normal histological structure of the glomeruli (G) and tubules (T). Results of H\&E staining in the UUO 14d group showed severe tubular atrophy and dilation, infiltration of inflammatory cells, and interstitial fibrosis. These findings were less pronounced in the UUO $3 \mathrm{~d}$ and UUO $7 \mathrm{~d}$ groups. In addition, Masson's staining of kidney tissues (Fig. 3B) in the sham group showed fine collagenic fibers between the renal tubules and around the blood vessels (arrow). Contrarily, in the UUO 14d group Masson's stain showed renal corpuscle and renal tubules undergo cystic dilatation (S) and collapsing (A). Furthermore, the collagenic fibers were massively condensed between renal tubules (arrow). These findings were less pronounced in the UUO $3 \mathrm{~d}$ and UUO 7d groups. 

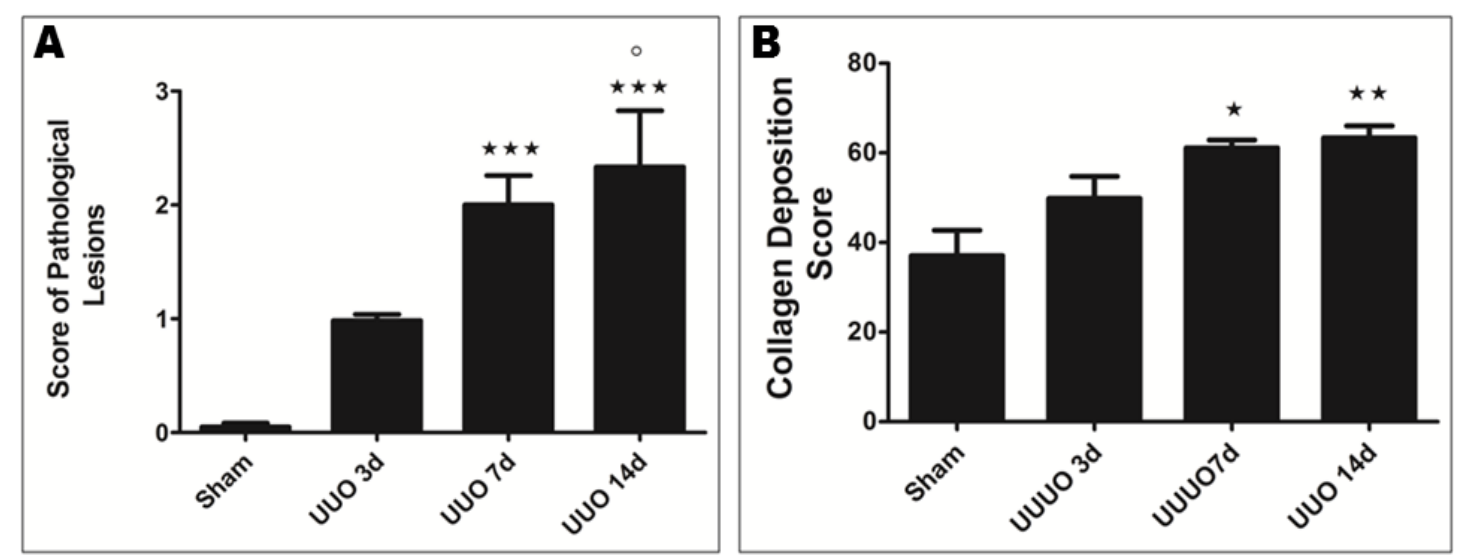

Figure 4: Score of histopathological changes (Fig. 4A) and the percentage of area occupied by Masson's staining (Fig. 4B) .Data are presented as mean \pm SEM $(n=6)$. *, and $\odot$ indicate significant change from sham, and UUO $3 \mathrm{~d}$ respectively. $*$, and $\circ$ indicate significant change at $\mathrm{p}<0.05 ; * *$, and $\circ \circ$ indicate significant change at $\mathrm{p}<$ $0.01 ; * *$, and $\circ \circ \circ$ indicate significant change at $\mathrm{p}<0.001$.

\subsection{Biochemical indices of renal performance}

Table 1 shows the concentrations of serum creatinine, BUN and microalbuminuria as classical indicators of renal function. Compared to sham group, the UUO groups exhibited a slight increase in creatinine, BUN and microalbuminuria concentration as early as 3 days after surgery, which remained increased on day 7 and day 14. A significant increase $(p<0.05)$ of both creatinine and microalbuminuria was observed only on day 14 compared with sham group.

Table 1 Biochemical indices of renal performance including serum creatinine, BUN and microalbuminuria.

\begin{tabular}{|c|c|c|c|}
\hline Group & $\begin{array}{c}\text { Creatinine } \\
(\boldsymbol{\mu m o l} / \mathbf{L})\end{array}$ & $\begin{array}{c}\text { BUN } \\
(\mathbf{m m o l} / \mathbf{L})\end{array}$ & $\begin{array}{c}\text { Microalbuminuria } \\
(\mathbf{m g} / \mathbf{L})\end{array}$ \\
\hline Sham & $26.33 \pm 1.89$ & $2.33 \pm 0.19$ & $18.00 \pm 1.46$ \\
\hline UUO 3d & $29.17 \pm 1.99$ & $2.48 \pm 0.22$ & $19.33 \pm 1.08$ \\
\hline UUO 7d & $31.50 \pm 2.05$ & $2.90 \pm 0.12$ & $20.50 \pm 1.23$ \\
\hline UUO 14d & $35.50 \pm 1.73^{*}$ & $2.97 \pm 0.17$ & $24.17 \pm 1.14^{*}$ \\
\hline
\end{tabular}

Data are presented as mean \pm SEM $(n=6) . *$ indicate significant change from sham operated group. $*$ indicate significant change at $\mathrm{p}<0.05$.

\subsection{Renal expression of TLR4, NF- $\kappa B$ and $\alpha-S M A$ after UUO}

Renal immune and non-immune cells express TLRs (Anders, 2010). But how are TLR4 and its downstream effector NF- $\kappa \mathrm{B}$ regulated in the postobstructive kidney? To address this question we performed UUO in 6-8 weeks old male albino mice, harvested kidneys at 3, 7 and 14 days after surgery, and performed western blot to assess protein levels expression in obstructed and sham operated kidneys. The renal protein levels of 
TLR4 and NF- $\kappa \mathrm{B}$ were over expressed in all obstructed but not in sham-operated kidneys from as early as 3 days after surgery and their expression level increased over time along with the known progressive nature of interstitial fibrosis after UUO (Figure $5 \mathrm{~A} \& \mathrm{~B})$. A similar protein expression pattern of an indicative marker for myofibroblasts activation $\alpha$-SMA was observed (Figure 5C).
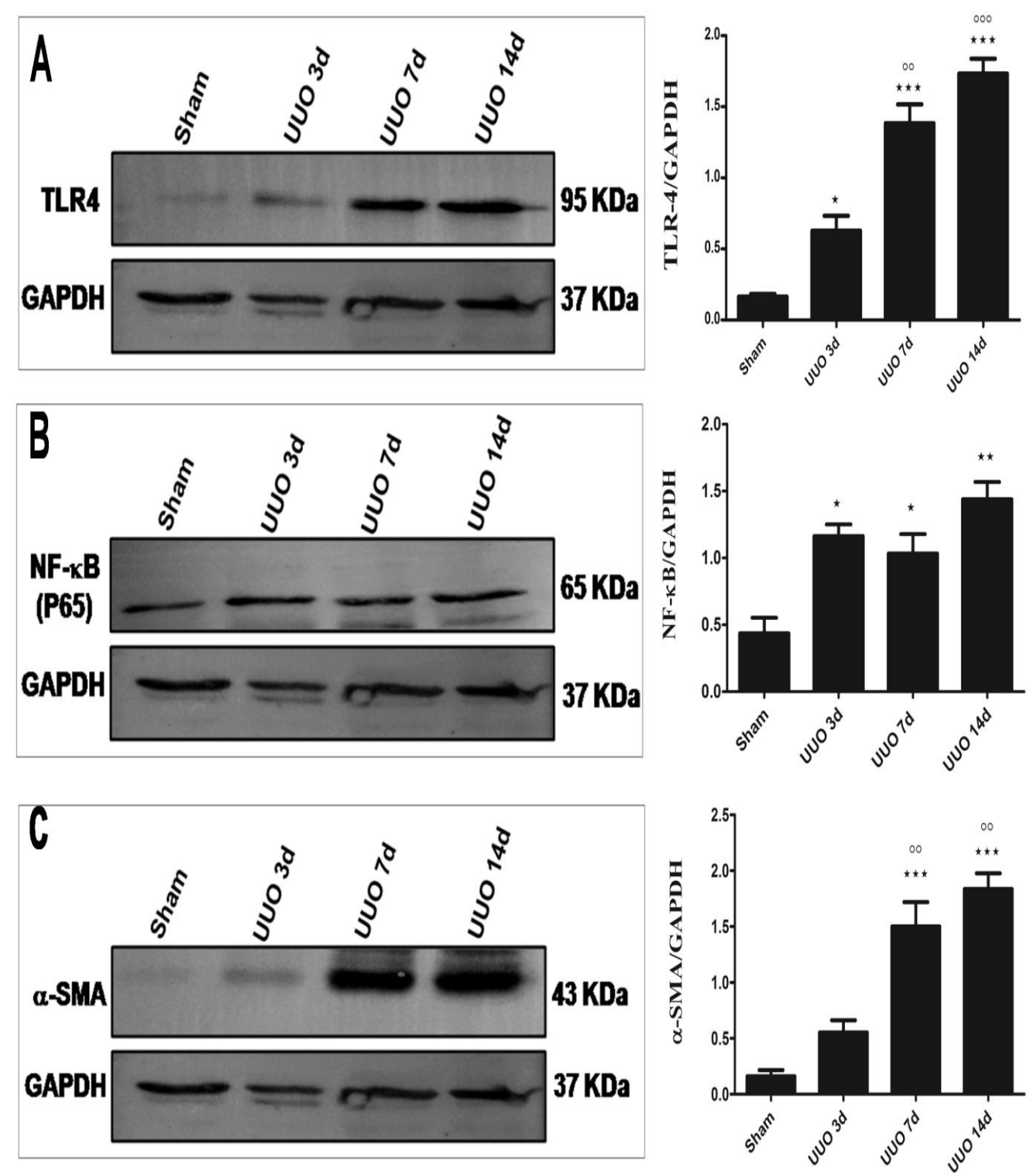

Figure 5: Representative analysis of western blotting and semiquantitative of average optical density of (A) TLR4, (B) NF- $\kappa B$ and (C) $\alpha$-SMA in renal tissue homogenates of different groups. Semiquantitative analysis is measured by Image $J^{\circledR}$ software and expressed as a percentage of GAPDH density. Data are presented as mean \pm SEM ( $\mathrm{n}=$ 3 ). $*$, and $\circ$ indicate significant change from sham and UUO $3 \mathrm{~d}$ respectively. ${ }^{*}$, and $\circ$ indicate significant change at $\mathrm{p}<0.05$; **, and $\circ \circ$ indicate significant change at $\mathrm{p}<$ $0.01 ; * * *$, and $\circ \circ \circ$ indicate significant change at $\mathrm{p}<0.001$. 


\section{Discussion}

In this study, we investigated the expression pattern of different inflammatory and fibrotic molecules which may affect fibrosis developments due to UUO. Our results revealed that progressive tubulointerstitial fibrosis and inflammation were increased as the UUO time increased.

Historically, surgical UUO in a variety of experimental animals has been used as a model of progressive chronic kidney injury. It is characterized by tubular atrophy, inflammation and interstitial fibrosis (Chevalier et al., 2009). UUO is interesting both as a model of ureteral obstruction and for studying the process of fibrosis as such (Ucero et al., 2014). In UUO, the primary damage is increased ureteral pressure transmitted retrograde to the kidney that leads to secondary renal vasoconstriction and resultant decreased glomerular blood flow (Duffield, 2014). In UUO mice model, the obstructed kidney develops extensive fibrosis over time. The increase in fibrosis is dependent on the time of UUO. Thus, the amount of collagen fibers increases by the time and the tissue architecture also undergoes gradually more changes (Ranjit $\boldsymbol{e t}$ al., 2016).

In order to evaluate the role of obstruction-induced tubulointerstitial fibrosis, renal samples were analyzed for evidence of collagen fibers accumulation. Our results demonstrated that the amount of collagen fibers increased over time and tissue architecture also undergoes progressively more changes. Two weeks of renal obstruction resulted in a significant increase in renal collagen fibers between renal tubules indicating an excessive fibrotic renal damage.

In fact, inflammatory and immunologic processes have been identified as an important pathogenic mechanism in several models of kidney injury, including drug nephrotoxicity, acute kidney injury and chronic obstructive damage. Thus, proinflammatory signaling pathways, soluble cytokines and chemokines, leukocyte cell subsets and endothelial activation, have been successfully targeted in experimental models to inhibit kidney injury and fibrosis progression (Sanz et al., 2010; Kitching and Holdsworth, 2011; Zafrani and Ince, 2015). Renal interstitial fibrosis typically resulted from chronic inflammation through released of related molecules, such as angiogenic factors, growth factors, fibrogenic cytokines and proteinase (Sutariya $\boldsymbol{e t}$ al., 2016). Indeed, renal inflammation is a universal response to infectious and noninfectious triggers. Noninfectious inflammation is an important component of various acute and chronic renal diseases (Segelmark and Hellmark, 2010; Park et al., 2014).

TLRs play a fundamental role in innate immune responses against invading microorganisms, but also promote sterile inflammation in a variety of diseases including acute kidney injury, atherosclerosis and diabetes (Anders and Schlondorff, 2007; Kaur et al., 2012). In vitro, exposure to high-concentration glucose caused TLR4 pathway activation culminating in NF-kB-dependent inflammatory responses in TECs and podocytes, and these responses were attenuated in cells genetically deficient in TLR4 (Mukherjee et al., 1958). 
TLR4 is present on kidney parenchymal cells, including podocytes, and the importance of TLR4-mediated parenchymal cell responses triggered by endogenous ligands is well recognized in other models of kidney injury, including ischemia reperfusion (Wu et al., 2007). Previous studies by Banas et al. reported that cultured podocytes constitutively express TLR4 and produce chemokines in response to stimulation with lipopolysaccharide (LPS), and that TLR4 expression by podocytes may be critical in triggering glomerular inflammation in a model of membranoproliferative glomerulonephritis (Banas et al., 2008).

Taken together the previous data, the present study, aimed to examine the expression pattern of TLR4 and $\mathrm{NF}-\kappa \mathrm{B}$ in different time grades of obstructive nephropathy which are presumed to be key regulators of renal fibrosis induced by UUO.

Results of the current study have established that there was over protein expression of TLR4 and its downstream modulator NF- $\kappa \mathrm{B}$ in all grades of renal fibrosis in comparison to the normal renal tissues with gradual increase from $3 \mathrm{~d}$ UUO model to reach its maximum in $14 \mathrm{~d}$ UUO model. The currently underlined steadily increasing TLR4 and NF- $\mathrm{BB}$ expression in renal tissues along with evidence of glomerular and tubular injury strongly supports a role for TLR4/NF- $\mathrm{B}$ pathway in mediating obstructive nephropathy. Our findings support earlier studies which suggested a role for TLR4 in the promotion of tubulo-interstitial inflammation in obstructive nephropathy (Pulskens et al., 2010; Lin et al., 2012).

Finally, the present study examined the tissue levels of $\alpha$-SMA as a marker of myofibroblasts proliferation and to clarify their link with TLR4 expression in the fibrotic renal tissue. Our current data demonstrated that the expression of $\alpha$-SMA as a fibrotic molecule was increased in UUO mouse kidney compared to sham kidney with gradual increase from $3 \mathrm{~d}$ UUO to reach its maximum in the late stage $14 \mathrm{~d}$ UUO model which is characterized by increased proliferation of myofibroblast and excessive extracellular matrix deposition and progressive fibrosis. The currently underlined steadily increasing $\alpha$-SMA expression in renal tissues inspires that $\alpha$-SMA might be a decisive event in the development and excessive synthesis of ECM that is maintained during fibrosis progression and myofibroblast cells proliferation. Renal fibroblast to myofibroblast phenoconversion is a key pathophysiological process because of the longterm negative consequences of chronic fibrosis in the kidney (Meran and Steadman, 2011). Our results are in accordance with previous studies (Tveitaras et al., 2015; Zhao et al., 2017). Interestingly, TLR4 signaling plays a key role during myofibroblast phenotypic transformation. Fibroblast proliferation was increased after myocardial infarction, and further phenotypic transformation of myofibroblasts is intensified following stimulation with LPS, a TLR4 ligand. Moreover, LPS was shown to induce $\alpha-$ SMA expression, in WT fibroblasts but did not affect TLR4 ${ }^{-/-}$fibroblast (Turner, 2016). These results suggest that TLR4 receptor is essential for facilitating the transformation of myofibroblasts.

\section{Conclusion}

In conclusion, this study confirmed previous reports which identified TLR4 as a mediator of inflammation in UUO, and also demonstrated its role in kidney fibrosis. We 
showed an association between TLR4, NF- $\kappa \mathrm{B}$ and $\alpha$-SMA expression and the development and severity of renal fibrosis in obstructed mice kidney. Given involvement of TLR4 pathways in these pivotal phases of obstructive nephropathy, strategies to inhibit TLR4 signaling should be explored

\section{Financial Disclosure}

This research did not receive any specific grant from funding agencies in the public, commercial, or not-for-profit sectors.

\section{Declaration of Interest Section}

The authors report no declarations of interest.

\section{REFERENCES}

Anders H. J. (2010): Toll-like receptors and danger signaling in kidney injury. J Am Soc Nephrol, 21(8): 1270-1274.

Anders H. J. and Schlondorff D. (2007): Toll-like receptors: emerging concepts in kidney disease. Curr Opin Nephrol Hypertens, 16(3): 177-183.

Banas M. C., Banas B., Hudkins K. L., Wietecha T. A., Iyoda M., Bock E., Hauser P., Pippin J. W., Shankland S. J., Smith K. D., et al. (2008): TLR4 links podocytes with the innate immune system to mediate glomerular injury. J Am Soc Nephrol, 19(4): 704-713.

Chevalier R. L., Forbes M. S. and Thornhill B. A. (2009): Ureteral obstruction as a model of renal interstitial fibrosis and obstructive nephropathy. Kidney Int, 75(11): 1145-1152.

Duffield J. S. (2014): Cellular and molecular mechanisms in kidney fibrosis. J Clin Invest, 124(6): 2299-2306.

Harmoinen A., Vuorinen P. and Jokela H. (1987): Turbidimetric measurement of microalbuminuria. Clin Chim Acta, 166(1): 85-89.

Kaur H., Chien A. and Jialal I. (2012): Hyperglycemia induces Toll like receptor 4 expression and activity in mouse mesangial cells: relevance to diabetic nephropathy. Am J Physiol Renal Physiol, 303(8): F1145-1150.

Kitching A. R. and Holdsworth S. R. (2011): The emergence of TH17 cells as effectors of renal injury. J Am Soc Nephrol, 22(2): 235-238.

Leemans J. C., Stokman G., Claessen N., Rouschop K. M., Teske G. J., Kirschning C. J., Akira S., van der Poll T., Weening J. J. and Florquin S. (2005): Renalassociated TLR2 mediates ischemia/reperfusion injury in the kidney. J Clin Invest, 115(10): 2894-2903. 
Lin M., Yiu W. H., Wu H. J., Chan L. Y., Leung J. C., Au W. S., Chan K. W., Lai K. N. and Tang S. C. (2012): Toll-like receptor 4 promotes tubular inflammation in diabetic nephropathy. J Am Soc Nephrol, 23(1): 86-102.

Liu B. C., Tang T. T. and Lv L. L. (2019): How Tubular Epithelial Cell Injury Contributes to Renal Fibrosis. Adv Exp Med Biol, 1165: 233-252.

Meran S. and Steadman R. (2011): Fibroblasts and myofibroblasts in renal fibrosis. International journal of experimental pathology, 92(3): 158-167.

Mora C. and Navarro J. F. (2006): Inflammation and diabetic nephropathy. Curr Diab Rep, 6(6): 463-468.

Mukherjee S., Kunitake G. and Alfinslater R. B. (1958): The esterification of cholesterol with palmitic acid by rat liver homogenates. J Biol Chem, 230(1): 91-96.

Nogueira A., Pires M. J. and Oliveira P. A. (2017): Pathophysiological Mechanisms of Renal Fibrosis: A Review of Animal Models and Therapeutic Strategies. In Vivo, 31(1): 1-22.

Page D. L. (1983): Theory and practice of histological techniques. Human Pathology, 14(10): 925-926.

Park J. S., Kim S., Jo C. H., Oh I. H. and Kim G. H. (2014): Effects of dietary salt restriction on renal progression and interstitial fibrosis in adriamycin nephrosis. Kidney Blood Press Res, 39(1): 86-96.

Peake M. and Whiting M. (2006): Measurement of serum creatinine--current status and future goals. Clin Biochem Rev, 27(4): 173-184.

Pulskens W. P., Rampanelli E., Teske G. J., Butter L. M., Claessen N., Luirink I. K., van der Poll T., Florquin S. and Leemans J. C. (2010): TLR4 promotes fibrosis but attenuates tubular damage in progressive renal injury. J Am Soc Nephrol, 21(8): 1299-1308.

Ranjit S., Dvornikov A., Levi M., Furgeson S. and Gratton E. (2016): Characterizing fibrosis in UUO mice model using multiparametric analysis of phasor distribution from FLIM images. Biomed Opt Express, 7(9): 3519-3530.

Sanz A. B., Sanchez-Nino M. D., Ramos A. M., Moreno J. A., Santamaria B., RuizOrtega M., Egido J. and Ortiz A. (2010): NF-kappaB in renal inflammation. J Am Soc Nephrol, 21(8): 1254-1262.

Schaefer L., Babelova A., Kiss E., Hausser H. J., Baliova M., Krzyzankova M., Marsche G., Young M. F., Mihalik D., Gotte M., et al. (2005): The matrix component biglycan is proinflammatory and signals through Toll-like receptors 4 and 2 in macrophages. J Clin Invest, 115(8): 2223-2233. 
Segelmark M. and Hellmark T. (2010): Autoimmune kidney diseases. Autoimmun Rev, 9(5): A366-371.

Shankar A., Sun L., Klein B. E., Lee K. E., Muntner P., Nieto F. J., Tsai M. Y., Cruickshanks K. J., Schubert C. R., Brazy P. C., et al. (2011): Markers of inflammation predict the long-term risk of developing chronic kidney disease: a population-based cohort study. Kidney Int, 80(11): 1231-1238.

Silverstein D. M. (2009): Inflammation in chronic kidney disease: role in the progression of renal and cardiovascular disease. Pediatr Nephrol, 24(8): 14451452.

Sutariya B., Jhonsa D. and Saraf M. N. (2016): TGF-beta: the connecting link between nephropathy and fibrosis. Immunopharmacol Immunotoxicol, 38(1): $39-49$.

Tabacco A., Meiattini F., Moda E. and Tarli P. (1979): Simplified enzymic/colorimetric serum urea nitrogen determination. Clin Chem, 25(2): 336-337.

Turner N. A. (2016): Inflammatory and fibrotic responses of cardiac fibroblasts to myocardial damage associated molecular patterns (DAMPs). J Mol Cell Cardiol, 94: 189-200.

Tveitaras M. K., Skogstrand T., Leh S., Helle F., Iversen B. M., Chatziantoniou C., Reed R. K. and Hultstrom M. (2015): Matrix Metalloproteinase-2 Knockout and Heterozygote Mice Are Protected from Hydronephrosis and Kidney Fibrosis after Unilateral Ureteral Obstruction. PLoS One, 10(12): e0143390.

Ucero A. C., Benito-Martin A., Izquierdo M. C., Sanchez-Nino M. D., Sanz A. B., Ramos A. M., Berzal S., Ruiz-Ortega M., Egido J. and Ortiz A. (2014): Unilateral ureteral obstruction: beyond obstruction. Int Urol Nephrol, 46(4): 765-776.

Vabulas R. M., Ahmad-Nejad P., Ghose S., Kirschning C. J., Issels R. D. and Wagner H. (2002): HSP70 as endogenous stimulus of the Toll/interleukin-1 receptor signal pathway. J Biol Chem, 277(17): 15107-15112.

Webster A. C., Nagler E. V., Morton R. L. and Masson P. (2017): Chronic Kidney Disease. Lancet, 389(10075): 1238-1252.

Wu H., Chen G., Wyburn K. R., Yin J., Bertolino P., Eris J. M., Alexander S. I., Sharland A. F. and Chadban S. J. (2007): TLR4 activation mediates kidney ischemia/reperfusion injury. J Clin Invest, 117(10): 2847-2859.

Xu Y., Ruan S., Wu X., Chen H., Zheng K. and Fu B. (2013): Autophagy and apoptosis in tubular cells following unilateral ureteral obstruction are associated with mitochondrial oxidative stress. Int J Mol Med, 31(3): 628-636. 
Yamaguchi J., Tanaka T. and Nangaku M. (2015): Recent advances in understanding of chronic kidney disease. F1000Res, 4.

Yu M., Wang H., Ding A., Golenbock D. T., Latz E., Czura C. J., Fenton M. J., Tracey K. J. and Yang H. (2006): HMGB1 signals through toll-like receptor (TLR) 4 and TLR2. Shock, 26(2): 174-179.

Zafrani L. and Ince C. (2015): Microcirculation in Acute and Chronic Kidney Diseases. Am J Kidney Dis, 66(6): 1083-1094.

Zeisberg M. and Neilson E. G. (2010): Mechanisms of tubulointerstitial fibrosis. J Am Soc Nephrol, 21(11): 1819-1834.

Zhao Y., Qiao X., Tan T. K., Zhao H., Zhang Y., Liu L., Zhang J., Wang L., Cao Q., Wang Y., et al. (2017): Matrix metalloproteinase 9-dependent Notch signaling contributes to kidney fibrosis through peritubular endothelialmesenchymal transition. Nephrol Dial Transplant, 32(5): 781-791. 


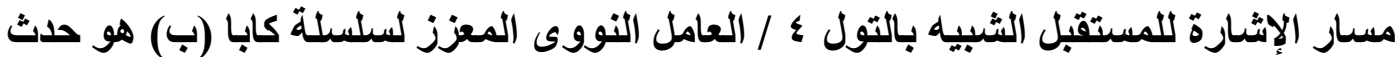

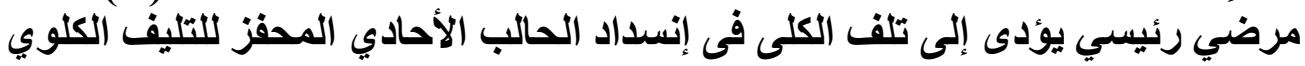

\author{
*عمرو مبروك توفيق' ، محمد أنور عبد العزيز' ، ثريا سعيد الديب ، عبد الرازق هاثم عبد الرازق ، ، بخيت

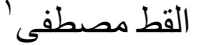 \\ 'قسم الكيمياء الحيوية_ كلية الصيدلة - جامعة الأزهر فرع أسيوط - أسيوط - جمهورية مصر العربية \\ 'قتم الكيمياء الحيوية ـ كلية الطب - جامعة أسيوط ـ أسيوط - جمهورية مصر العربية \\ "َّم الهستولوجى - كلية الطب البيطرى - جامعة بنى سويف - بنى سويف ـ جمهورية مصر العربية \\ amrallam@azhar.edu.eg : البريد الاكتروني للباحث الرئيسي *
}

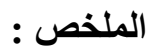

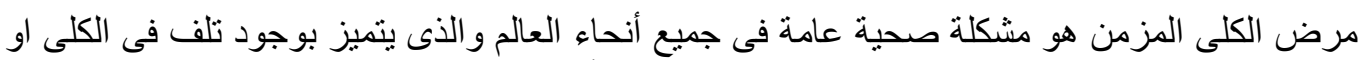

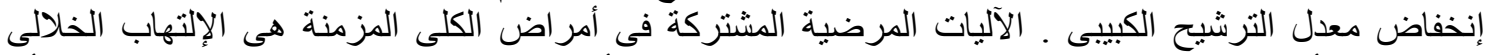

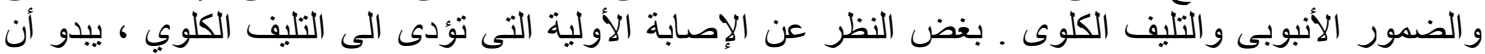

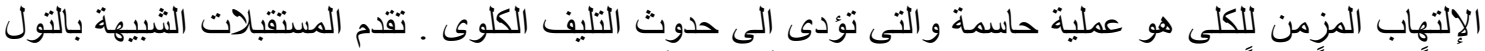

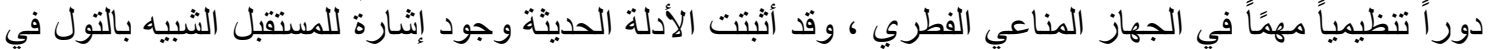

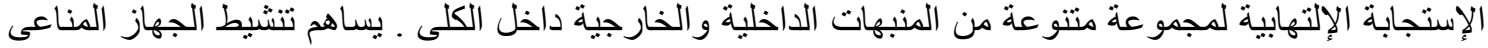

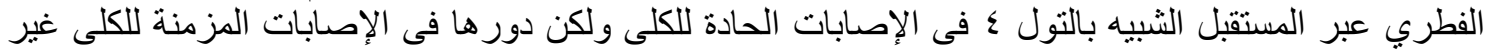

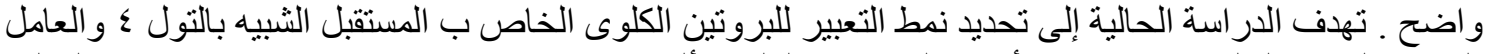

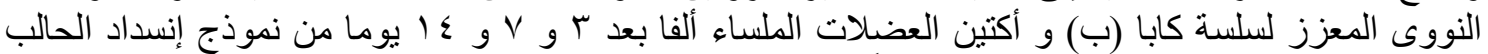

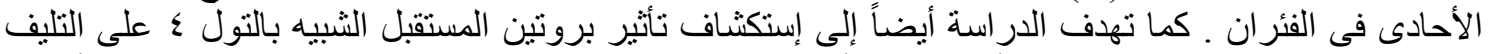

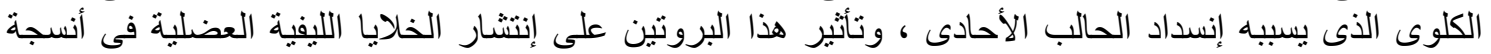

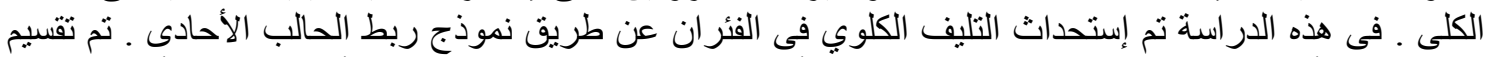

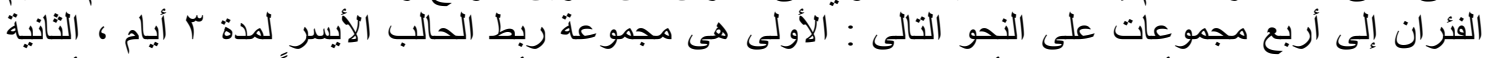

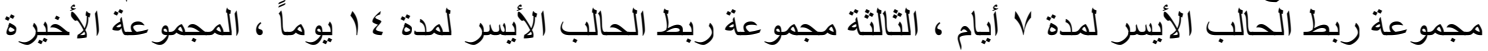

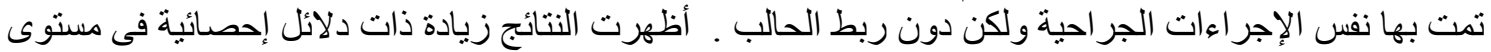

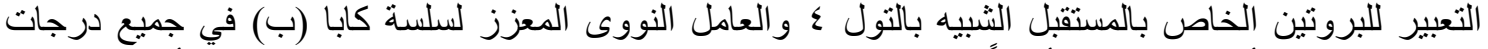

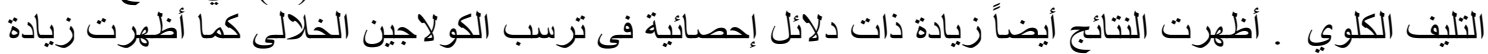

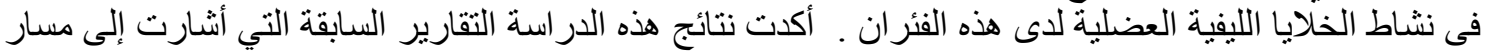

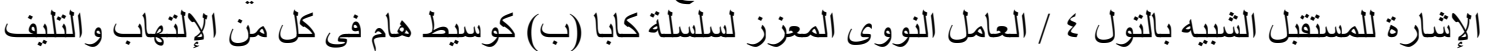

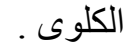

الكلمات المفتاحية : حصيلة مثل مستقبلات. العامل النووي كابا ب. التهاب؛ الكلى؛ انسداد الحالب من جانب واحد. ألفا العضلات الملساء الأكتين. تليف. 